\title{
A Technique for Constrained Optimization of Cross-ply Laminates using a New Variant of Genetic Algorithm
}

\author{
Huiyao Zhang ${ }^{1}$, Atsushi Yokoyama ${ }^{2}$ \\ Department of Fiber Science and Engineering \\ Kyoto Institute of Technology, \\ Kyoto, JAPAN
}

\begin{abstract}
The main challenge presented by the design of laminated composite material is the laminate layup, involving a set of fiber orientations, composite material systems, and stacking sequences. In nature, it is a combinatorial optimization problem with constraints that can be solved by the genetic algorithm. The traditional approach to solve a constrained problem is reformulating the objective function. In the present study, a new variant of the genetic algorithm is proposed for the design of composite material by using a mix of selection strategies, instead of modifying the objective function. To check the feasibility of a laminate subject to in-plane loading, the effect of the fiber orientation angles and material components on the first ply failure is studied. The algorithm has been validated by successfully optimizing the design of cross-ply laminate under different inplane loading cases. The results obtained by this algorithm are better than works in related literature.
\end{abstract}

Keywords-Laminated composite; classical lamination theory; genetic algorithm; optimal design

\section{INTRODUCTION}

Composite materials offer improved strength, stiffness, fatigue, corrosion resistance, etc. over conventional materials, and are widely used as materials for applications ranging from the automotive to shipbuilding industry, electronic packaging to golf clubs, and medical equipment to homebuilding. However, the high cost of fabrication of composites is a critical drawback to its application. For example, the graphite/epoxy composite part may cost as much as 650 to 900 per kilogram. In contrast, the price of glass/epoxy is about 2.5 times less. Manufacturing techniques such as sheet molding compounds and structural reinforcement injection molding are used to lower the costs for manufacturing automobile parts. An alternative approach is using hybrid composite materials.

The mechanical performance of a laminate composite is affected by a wide range of factors such as the thickness, material, and orientation of each lamina. Because of manufacturing limitations, all these variables are usually limited to a small set of discrete values. For example, the ply thickness is fixed, and ply orientation angles are limited to a set of angles such as 0 , 45 , and 90 degrees in practice. So the search process for the optimal design is a discrete optimization problem that can be solved by the GA. To tailor a laminate composite, the GA has been successfully applied to solve laminate design problems [1], [2], [3], [4], [5], [6], [7], [8], [9], [10], [11]. The GA simulates the process of natural evolution, including selection, crossover, and mutation according to Darwin's principle of "survival of the fittest". The known advantages of GAs are the following: (i) GAs are not easily trapped in local optima and can obtain the global optimum. (ii) GAs do not need gradient information and can be applied to discrete optimization problems. (iii) GAs can not only find the optimal value in the domain but also maintain a set of optimal solutions. However, the GA also has some disadvantages, for example, the GA needs to evaluate the target functions many times to achieve optimization, and the cost of the search process is high. The GA consists of some basic parts, the coding of the design variable, the selection strategy, the crossover operator, the mutation operator, and how to deal with constraints. For the variable design part, there are two methods to deal with the representation of design variables, namely, binary string and real value representation [1], [4], [12], [13]. Michalewicz [14] claimed that the performance of floating-point representation was better than binary representation in the numerical optimization problem. Selection strategy plays a critical role in the GA, which determines the convergence speed and the diversity of the population. To improve search ability and reduce search costs, various selection methods have been invented, and they can be divided into four classes: proportionate reproduction, ranking, tournament, and genitor(or "steady state") selection. In the optimization of laminate composite design, the roulette wheel [1], [15], where the possibility of an individual to be chosen for the next generation is proportional to the fitness. Soremekun et al. [16] showed that the generalized elitist strategy outperformed a single individual elitism in some special cases.

The data structure, repair strategies, and penalty functions [17] are the most commonly used approaches to resolve constrained problems in the optimization of composite structures. Symmetric laminates are widely used in practical scenarios, and data structures can be used to fulfill symmetry constraints, which consists of coding half of the laminate and considering the rest with the opposite orientation. Todoroki [4] introduced a repair strategy that can scan the chromosome and repair the gene on the chromosome if it does not satisfy the contiguity constraint. The comparison of repair strategies in a permutation GA with the same orientation was presented by Liu et al. [5], and it showed that the Baldwinian repair strategy can substantially reduce the cost of constrained optimization. Haftka and Todoroki [1] used the GA to solve the laminate stacking sequence problem using a penalty function subject to buckling and strength constraints.

In typical engineering applications, composite materials are under very complicated loading conditions, not only in-plane loading but also out-of-plane loading. Most of the studies on the optimization of the laminate composite material minimized the thickness [18], [7], weight [19], [20], [21], and cost and weight [20], [22], or maximized the static strength of the composite laminates for a targeted thickness [7], [8], [23], 
[24]. In the present study, the cost and weight of laminates are minimized by modifying the objective function.

To check the feasibility of a laminate composite by imposing a strength constraint, failure analysis of a laminate is performed by applying suitable failure criteria. The failure criteria of laminated composites can be classified into three classes: non-interactive theories (e.g. maximum strain), interactive theories (e.g. Tsai-wu), and partially interactive theories (e.g. Puck failure criterion). Previous researchers adopted the first-ply-failure approach using Tsai-wu failure theory [25], [26], [19], [27], [28], [29], [22], [30], Tsai-Hill [31], [32], the maximum stress [33], or the maximum strain[33] static failure criteria. Akbulut [11] used the GA to minimize the thickness of composite laminates with Tsai-Hill and maximum stress failure criteria, and the advantage of this method is it avoids spurious optima. Naik et al. [34] minimized the weight of laminated composites under restrictions with a failure mechanism-based criterion based on the maximum strain and Tsai-wu. In the present study, Tsai-wu Static failure criteria are used to investigate the feasibility of a laminate composite.

\section{Classical Lamination Theory}

A laminate structure consists of multiple laminae bonded together through their thickness. Considering a laminate composite plate that is subject to in-plane loading of extension, shear, bending, and torsion, the classical lamination theory (CLT) is taken to calculate the stress and strain in the local and global axes of each ply, as shown in Fig. 1. Based on fiber orientation, material, and fiber thickness, there are a few special cases of laminate: the set of fiber angles in Fig. 2 only includes 0 and 90, which is called cross-ply laminate.

\section{A. Stress and Strain in Lamina}

For a single lamina, the stress-strain relation in local axis 1-2 is:

$$
\left[\begin{array}{c}
\sigma_{1} \\
\sigma_{2} \\
\tau_{12}
\end{array}\right]=\left[\begin{array}{ccc}
Q_{11} & Q_{12} & 0 \\
Q_{12} & Q_{22} & 0 \\
0 & 0 & Q_{66}
\end{array}\right]\left[\begin{array}{c}
\varepsilon_{1} \\
\varepsilon_{2} \\
\gamma_{12}
\end{array}\right],
$$

where $Q_{i j}$ are the stiffnesses of the lamina that are related

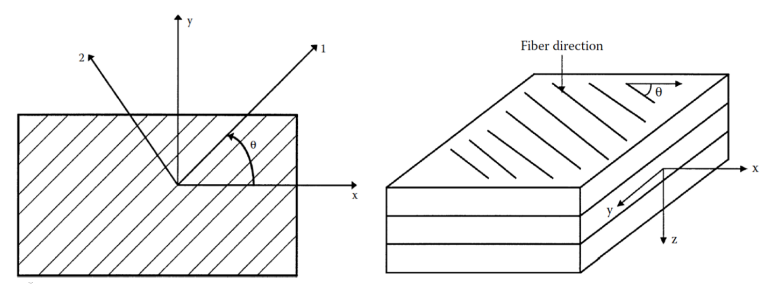

Fig. 1. Local and Global Axes of an Angle Lamina.

\begin{tabular}{c}
\hline 0 \\
\hline 90 \\
\hline$\cdots$ \\
\hline 0 \\
\hline 90 \\
\hline
\end{tabular}

Fig. 2. Model for Cross-ply Laminate. to engineering elastic constants given by

$$
\begin{aligned}
Q_{11} & =\frac{E_{1}}{1-v_{12} v_{21}}, \\
Q_{22} & =\frac{E_{2}}{1-v_{12} v_{21}}, \\
Q_{66} & =G_{12}, \\
Q_{12} & =\frac{v_{21} E_{2}}{1-v_{12} v_{21}},
\end{aligned}
$$

where $E_{1}, E_{2}, v_{12}, G_{12}$ are four independent engineering elastic constants, which are defined as follows: $E_{1}$ is the longitudinal Young's modulus, $E_{2}$ is the transverse Young's modulus, $v_{12}$ is the major Poisson's ratio, and $G_{12}$ is the inplane shear modulus.

Stress strain relation in the global x-y axis:

$$
\left[\begin{array}{l}
\sigma_{x} \\
\sigma_{y} \\
\tau_{x y}
\end{array}\right]=\left[\begin{array}{lll}
\bar{Q}_{11} & \bar{Q}_{12} & \bar{Q}_{16} \\
\bar{Q}_{12} & \bar{Q}_{22} & \bar{Q}_{26} \\
\bar{Q}_{16} & \bar{Q}_{26} & \bar{Q}_{66}
\end{array}\right]\left[\begin{array}{l}
\varepsilon_{x} \\
\varepsilon_{y} \\
\gamma_{x y}
\end{array}\right],
$$

where

$$
\begin{aligned}
& \bar{Q}_{11}=Q_{11} c^{4}+Q_{22} s^{4}+2\left(Q_{12}+2 Q_{66}\right) s^{2} c^{2}, \\
& \bar{Q}_{12}=\left(Q_{11}+Q_{22}-4 Q_{66}\right) s^{2} c^{2}+Q_{12}\left(c^{4}+s^{2}\right), \\
& \bar{Q}_{22}=Q_{11} s^{4}+Q_{22} c^{4}+2\left(Q_{12}+2 Q_{66}\right) s^{2} c^{2}, \\
& \bar{Q}_{16}=\left(Q_{11}-Q_{12}-2 Q_{66}\right) c^{3} s-\left(Q_{22}-Q_{12}-2 Q_{66}\right) s^{3} c, \\
& \bar{Q}_{26}=\left(Q_{11}-Q_{12}-2 Q_{66}\right) c s^{3}-\left(Q_{22}-Q_{12}-2 Q_{66}\right) c^{3} s, \\
& \bar{Q}_{66}=\left(Q_{11}+Q_{22}-2 Q_{12}-2 Q_{66}\right) s^{2} c^{2}+Q_{66}\left(s^{4}+c^{4}\right) .
\end{aligned}
$$

The $\mathrm{c}$ and $\mathrm{s}$ denote $\cos \theta$ and $\sin \theta$, respectively.

The local and global stresses in an angle lamina are related to each other through the angle of the lamina $\theta$

$$
\left[\begin{array}{l}
\sigma_{1} \\
\sigma_{2} \\
\tau_{12}
\end{array}\right]=[T]\left[\begin{array}{l}
\sigma_{x} \\
\sigma_{y} \\
\tau_{x y}
\end{array}\right]
$$

where

$$
[T]=\left[\begin{array}{ccc}
c^{2} & s^{2} & 2 s c \\
s^{2} & c^{2} & -2 s c \\
-s c & s c & c^{2}-s^{2}
\end{array}\right]
$$

B. Stress and Strain in a Laminate

$$
\begin{aligned}
& {\left[\begin{array}{c}
N_{x} \\
N_{y} \\
N_{x y}
\end{array}\right]=} {\left[\begin{array}{lll}
A_{11} & A_{12} & A_{16} \\
A_{12} & A_{22} & A_{26} \\
A_{16} & A_{26} & A_{66}
\end{array}\right]\left[\begin{array}{c}
\varepsilon_{x}^{0} \\
\varepsilon_{y}^{0} \\
\gamma_{x y}^{0}
\end{array}\right] } \\
&+ {\left[\begin{array}{lll}
B_{11} & B_{12} & B_{16} \\
B_{11} & B_{12} & B_{16} \\
B_{16} & B_{26} & B_{66}
\end{array}\right]\left[\begin{array}{c}
k_{x} \\
k_{y} \\
k_{x y}
\end{array}\right] } \\
& {\left[\begin{array}{c}
M_{x} \\
M_{y} \\
M_{x y}
\end{array}\right]=\left[\begin{array}{lll}
B_{11} & B_{12} & B_{16} \\
B_{12} & B_{22} & B_{26} \\
B_{16} & B_{26} & B_{66}
\end{array}\right]\left[\begin{array}{c}
\varepsilon_{x}^{0} \\
\varepsilon_{y}^{0} \\
\gamma_{x y}^{0}
\end{array}\right] } \\
&+\left[\begin{array}{lll}
D_{11} & D_{12} & D_{16} \\
D_{11} & D_{12} & D_{16} \\
D_{16} & D_{26} & D_{66}
\end{array}\right]\left[\begin{array}{c}
k_{x} \\
k_{y} \\
k_{x y}
\end{array}\right]
\end{aligned}
$$

$N_{x}, N_{y}$ - normal force per unit length 
$N_{x y}$ - shear force per unit length

$M_{x}, M_{y}$ - bending moment per unit length

$M_{x y}$ - twisting moments per unit length

$\varepsilon^{0}, k$ - mid-plane strains and curvature of a laminate in $\mathrm{x}-\mathrm{y}$ coordinates

The mid-plane strain and curvature is given by

$$
\begin{aligned}
& A_{i j}=\sum_{k=1}^{n}\left(\overline{Q_{i j}}\right)_{k}\left(h_{k}-h_{k-1}\right) i=1,2,6, j=1,2,6, \\
& B_{i j}=\frac{1}{2} \sum_{k=1}^{n}\left(\overline{Q_{i j}}\right)_{k}\left(h_{k}^{2}-h_{k-1}^{2}\right) i=1,2,6, j=1,2,6, \\
& D_{i j}=\frac{1}{3} \sum_{k=1}^{n}\left(\overline{Q_{i j}}\right)_{k}\left(h_{k}^{3}-h_{k-1}^{3}\right) i=1,2,6, j=1,2,6,
\end{aligned}
$$

where the $[\mathrm{A}],[\mathrm{B}]$, and $[\mathrm{D}]$ matrices are called the extensional, coupling, and bending stiffness matrices.

\section{FAILURE THEORY}

\section{A. Failure Process}

A laminate will fail under increasing mechanical loading; however, the procedure of laminate failure may not be catastrophic. In some cases, some layers fail first, and the rest are able to continue to take additional loading until all the plies fail. A ply is fully discounted when a ply fails; then, the ply is replaced by a near-zero stiffness and strength. The procedure for finding the first ply failure in the present study follows the fully discounted method:

1) Compute the reduced stiffness matrix [Q] referred to as the local axis for each ply using its four engineering elastic constants $E_{1}, E_{2}, E_{12}$, and $G_{12}$.

2) Calculate the transformed reduced stiffness $[\bar{Q}]$ referring to the global coordinate system $(\mathrm{x}, \mathrm{y})$ using the reduced stiffness matrix [Q] obtained in step 1 and the ply angle for each layer.

3) Given the thickness and location of each layer, the three laminate stiffness matrices $[\mathrm{A}],[\mathrm{B}]$, and [D] are determined.

4) Apply the forces and moments, $[N]_{x y},[M]_{x y}$ solve Equation 7, and calculate the middle plane strain $\left[\sigma^{0}\right]_{x y}$ and curvature $[k]_{x y}$.

5) Determine the local strain and stress of each layer under the applied load.

6) Use the ply-by-ply stress-strain and related failure theories to determine the strength ratio.

\section{B. Tsai-wu Failure Theory}

Many different theories about the failure of an angle lamina have been developed for a unidirectional lamina, such as the maximum stress failure theory, maximum strain failure theory, Tsai-Hill failure theory, and Tsai-Wu failure theory. The failure theories of a lamina are based on the stresses in the local axes in the material. There are four normal strength parameters and one shear stress for a unidirectional lamina. The five strength parameters are:

$$
\begin{aligned}
& \left(\sigma_{1}^{T}\right)_{\text {ult }}=\text { ultimate longitudinal tensile strength } \\
& \left(\sigma_{1}^{C}\right)_{\text {ult }}=\text { ultimate longitudinal compressive strength }
\end{aligned}
$$

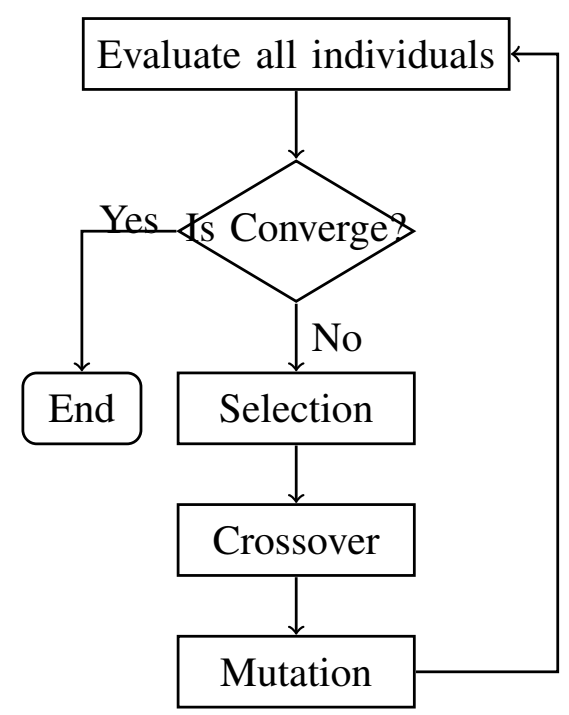

Fig. 3. Traditional GA Model.

$\left(\sigma_{2}^{T}\right)_{\text {ult }}=$ ultimate transverse tensile strength

$\left(\sigma_{2}^{C}\right)_{u l t}=$ ultimate transverse compressive strength

$\left(\tau_{12}\right)_{u l t}=$ and ultimate in-plane shear strength

In the present study, Tsai-wu failure theory is taken to decide whether a lamina fails, because this theory is more general than the Tsai-Hill failure theory, which considers two different situations, the compression and tensile strengths of a lamina. A lamina is considered to fail if

$$
\begin{aligned}
H_{1} \sigma_{1} & +H_{2} \sigma_{2}+H_{6} \tau_{12}+H_{11} \sigma_{1}^{2}+H_{22} \sigma_{2}^{2} \\
& +H_{66} \tau_{12}^{2}+2 H_{12} \sigma_{1} \sigma_{2}<1
\end{aligned}
$$

is violated, where

$$
S R=\frac{\text { Maximum Load }}{\text { Load Applied }}
$$

The maximum load refers to that can be applied using Tsaiwu failure theory.

\section{Genetic Algorithm Model}

The genetic algorithm starts with multiple individuals with limited chromosome lengths, in which maybe none of these individuals fulfill the constraints. The GA is assumed to derive appropriate offspring based on the initial population as the GA continues. The traditional way to handle the constrained search of the GA is either to introduce repair strategies or to use a penalty function. Fig. 3 shows the classic flow chart of a GA framework, which includes selection, crossover, and mutation operators. However, GA is originally proposed to solve unconstrained problems; therefore, we suggest a new approach to address the constrained GA search problem in an unconstrained way.

Because of the existence of constraints, the population not only can be sorted by the fitness (obtained by the objective function) but also sorted by the constraint value obtained by the constraint function (assuming a constraint function exists), so the parents of the next generation can be chosen by the 
following three approaches. First, the population is sorted by fitness in ascending order, and individuals with smaller fitness are selected. These selected individuals form a group named as a proper group. Second, remove individual which satisfies constraints, and sort population by the difference between the individual's constraint value and the threshold of the constraint in descending order, and individuals with bigger differences are chosen to be the parents of the next generation. The group which forms are called potential group, and an individual from this group is referred to as a potential individual. Third, the population is sorted by fitness from low to high after removing individuals which fail to fit the constraints, select individuals with bigger fitness, and these individuals form the proper group. So the final parents' pool is consists of three groups, active group, potential group, and proper group. The number of active individuals, potential individuals, and proper individuals are called, respectively, active number, potential numbers, and proper number.

Each group in the parents' population has its role in the searching process. The problem within traditional GA is premature and has weak local search ability, therefore, traditional GAs are more likely to get stuck in a local optimum. To prevent the GA from experiencing early convergence and to improve the local search performance, the active group is proposed to overcome this problem. As its name suggests, this group would always live in the population. Because both active individual's fitness and constraint value are small, each individual can be treated as an independent gene clip. So their existence enriches the gene clip variety of the mating pool. The offspring of two active individuals are more likely to be active individuals, which can maintain the active group.

For an individual in the potential group, it doesn't satisfy the constraint, however, it's supposed to evolve into a proper individual after multiple generations by modifying its chromosome structure or length. The crossover operation could happen between a potential individual with an active individual, or a potential individual, or a proper individual. The child of an active individual and a potential individual is more likely to be a potential individual, and this active individual could inject a new gene clip into this potential individual, therefore providing a new evolution direction.

A proper individual means a feasible solution, which fulfills all the constraints. However, there are still some drawbacks within it, for example, its fitness is low. The crossover operation could happen between a proper individual and any other individuals.

The mutation operator for an active group is different from the potential group and proper group because their roles in the searching process are different: the target of the potential group and proper group is to obtain a feasible solution; however, the role of the active group is to maintain the variety of gene clips in the mating pool.

Fig. 4 shows the flow chart of the proposed GA. First, the population is divided into three groups, active group, potential group, and proper group by the above-mentioned method. Second, select an appropriate number of individuals from each group as parents, and the various selection scheme can be taken for each group.

The searching process can be divided into two phases according to whether proper individuals are generated or not. During the initial stage, no individual in the population is appropriate, which means the number of individuals in the proper group is zero. Both active group and potential group are full. After a couple of generations, some proper individuals could be produced. Then, the GA comes to its second phase, the number of proper individuals begins to increase, finally, the number in the proper group reaches its upper bound.

\section{EXPERIMENT}

First, we formulate a constrained problem by searching the optimal stacking sequence of cross-ply laminate under in-plane loading under the constraint whose strength ratio is not less than two. Each lamina dimensions $1000 \times 1000 \times$ $0.165 \mathrm{~mm}^{3}$ is adopted in this experiment, each graphite/epoxy, and glass/epoxy layer is assumed to cost 2.5 and 1 monetary unit, respectively. The other material properties are shown in Table I.

TABLE I. COMPARISON OF THE GRAPHITE/EPOXY AND GLASS/EPOXY PROPERTIES

\begin{tabular}{lcccc}
\hline Property & Symbol & Unit & Graphite/Epoxy & Glass/Epoxy \\
\hline Longitudinal elastic modulus & $E_{1}$ & $\mathrm{GPa}$ & 181 & 38.6 \\
Traverse elastic modulus & $E_{2}$ & $\mathrm{GPa}$ & 10.3 & 8.27 \\
Major Poisson's ratio & $v_{12}$ & & 0.28 & 0.26 \\
Shear modulus & $G_{12}$ & $\mathrm{GPa}$ & 7.17 & 4.14 \\
Ultimate longitudinal tensile strength & $\left(\sigma_{1}^{T}\right)_{u l t}$ & $\mathrm{MP}$ & 1500 & 1062 \\
Ultimate longitudinal compressive strength & $\left(\sigma_{1}^{C}\right)_{u l t}$ & $\mathrm{MP}$ & 1500 & 610 \\
Ultimate transverse tensile strength & $\left(\sigma_{2}^{T}\right)_{u l t}$ & $\mathrm{MPa}$ & 40 & 31 \\
Ultimate transverse compressive strength & $\left(\sigma_{2}^{C}\right)_{u l t}$ & $\mathrm{MPa}$ & 246 & 118 \\
Ultimate in-plane shear strength & $\left(\tau_{12}\right)_{u l t}$ & $\mathrm{MPa}$ & 68 & 72 \\
Density & $\rho$ & $g / \mathrm{cm}^{3}$ & 1.590 & 1.903 \\
Cost & & & 2.5 & 1 \\
\hline
\end{tabular}

\section{A. Problem Formulation}

In the present experiment, the optimal composite sequences, and the number of layers for a targeted strength

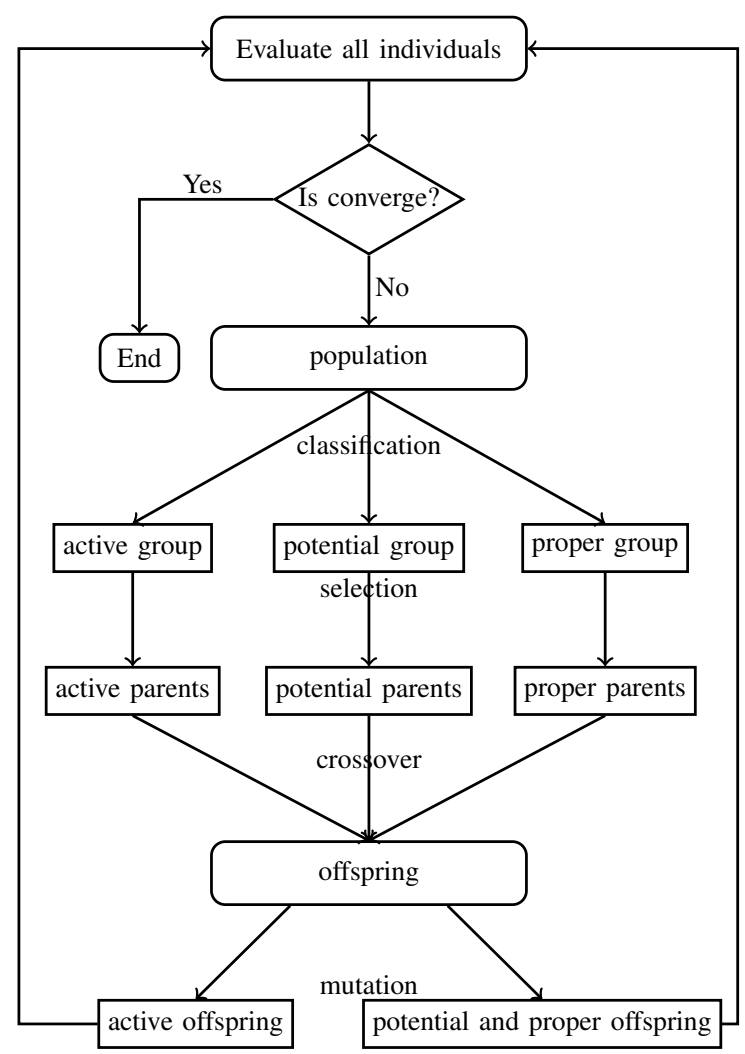

Fig. 4. General Flowchart of Proposed GA Model in which the Parents is Consist of three Different Groups. 
ratio under in-plane loading conditions are investigated. The aim is to minimize the mass of a laminate composite for a targeted strength ratio based on the Tsai-wu failure theory. The design variables are the ply angles and the number of layers. Ply orientation restricted to a discrete set of angles ( 0 , and 90 degrees). The problem can be formulated as the following equation:

Find: $\left\{\theta_{k}, n\right\} \theta_{k} \in\{0,90\}$,

Minimize: weight,

Subject to: strength ratio.

\section{B. GA Operation}

In the present study, floating encoding is adopted to represent a solution for the layup design of cross-ply laminate, Fig. 5(a) shows two parents $P_{1}$ and $P_{2}$ represent two cross-ply laminates, the corresponding laminates layups are $\left[0_{3} / 90_{7}\right]$ and $\left[0_{6} / 90_{4}\right]$, respectively; Fig. 5 (b) shows two offspring of parents $P_{1}$ and $P_{2}$ are consist of half of each parent's chromosome; Fig. 5(c) and 5(d) display the offspring after length and angle mutation, respectively.

For the length mutation of chromosome, calculate the chromosome's strength ratio based on its sequence, if its strength ratio is less than the threshold, then increase its length; otherwise, reduce it. We introduce the term length mutation coefficient to control the length mutation. As shown in Fig. 5 (b), the strength ratio of $O_{1}$ chromosome is 0.0854 , and the strength ratio threshold is five. Suppose the length mutation coefficient takes two, then the corresponding increase length is the multiplication result of length mutation coefficient and the difference between current strength ratio and the threshold: the result is $5 \times(2-0.0854)$, round this number to its closest integer, which is 9 . So the length of offspring $O_{1}$ changes from 10 to 19 after length mutation. For the angle mutation, randomly swap the gene from 0 to 90 in the chromosome, or the otherwise.

\begin{tabular}{|c|c|c|c|c|c|c|c|c|c|c|}
\hline$P_{1}$ & 90 & 90 & 0 & 0 & 0 & 90 & 90 & 90 & 90 & 90 \\
\hline$P_{2}$ : & 0 & 0 & 90 & 90 & 90 & 0 & 0 & 90 & 0 & 0 \\
\hline
\end{tabular}

(a): Parents $P_{1}$ and $P_{2}$

\begin{tabular}{l|c|c|c|c|c|c|c|c|c|c|}
$O_{1}:$ & 90 & 90 & 0 & 0 & 0 & 0 & 0 & 90 & 0 & 0 \\
\hline$O_{2}:$ & 0 & 0 & 90 & 90 & 90 & 90 & 90 & 90 & 90 & 90 \\
\hline
\end{tabular}

(b): Offspring $\mathrm{O}_{1}$ and $\mathrm{O}_{2}$

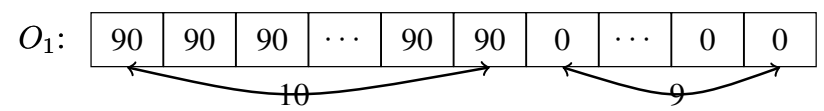

(c): Offspring $O_{1}$ after lenght mutation

\begin{tabular}{|l|l|l|l|l|l|l|l|l|l|}
\hline 90 & 90 & 90 & $\cdots$ & 90 & 0 & 0 & $\cdots$ & 0 & 0 \\
\hline
\end{tabular}

(d): Offspring $O_{1}$ after angle mutation

Fig. 5. Examples of Crossover, Length Mutation, Angle Mutation Operator for Proposed GA.

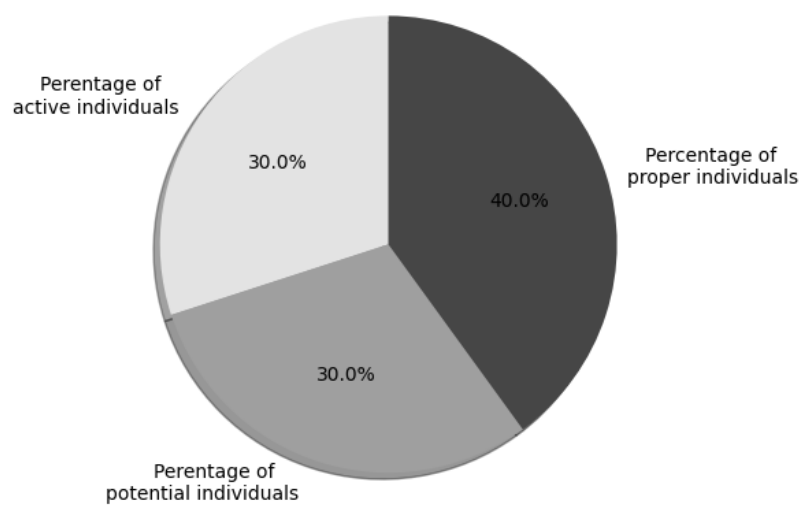

Fig. 6. Percentage of Active Individuals from Active Group, Potential Individuals from Potential Group, and Proper Individuals from Proper Group in Parent Population.

\section{GA Parameters}

Table II shows related GA parameters: the population is 40, and 50 percent is as the mating pool, so the parent population is 20; as shown in Fig. 6, the percentage of active individuals from the active group, potential individuals from the potential group, and proper individuals from the proper group are 0.3, 0.3 , and 0.4 , which means the corresponding number of these three types of individuals are 6, 6, and 8, respectively.

\section{NumericAl Results AND Discussion}

To figure out how the number of individuals in each group varies during the GA process, we conduct a onetime experiment and show the number of individuals in each generation with respect of GA generation. Second, to verify its performance and stability, the GA is run one hundred times: the best, worst case, and average results are presented, respectively. Finally, we compare the results with the work in the other literature.

TABLE II. PARAMETERS OF Proposed GA MOdel

\begin{tabular}{lc}
\hline Parameter & Value \\
\hline Population & 40 \\
Initial length range & {$[3-15]$} \\
Encoding & Integer \\
Percentage of parent & 0.5 \\
Percentage of active group & 0.3 \\
Percentage of potential group & 0.3 \\
Percentage of proper group & 0.4 \\
Selection strategy for active group & Ranking \\
Selection strategy for potential group & Ranking \\
Selection strategy for proper group & Ranking \\
Crossover strategy & One-point \\
Mutation strategy & Mass mutation \\
Length mutation coefficient & 5 \\
Angle mutation rate & 0.1 \\
Crossover rate & 0.3 \\
\hline
\end{tabular}


Fig. 7 shows the number of individuals in each group during the one-time GA process. For both active group and potential group, the number of individuals is fixed, and equal to its upper bound from the beginning to the end of the searching process. However, for the proper group, at the initial stage of GA, no individual fulfills the constraint, so the number of proper individuals is zero. As seen from Fig. 7, after forty generations, proper individuals appear, and its population increases very quickly up to its upper bound.

There are two approaches that the GA could obtain a better solution: the first is increasing the length of the chromosome; the second one is adjusting the internal structure of a chromosome. The GA process can be divided into two phases by whether there are proper individuals or not. Fig. 8 shows the GA process in which the dashed vertical line is the watershed between the initial phase and the last phase. In the initial phase, no individual's strength ratio is over the specified threshold, and the main reason that the fitness gets smaller gradually is the increase of chromosome's length; At point 1 on the fitness curve, the fitness suddenly goes up, however, the corresponding strength ratio of point 1 , denoted by the point $1^{\prime}$ on the generation-strength ratio curve, also increases. it is because of the adjustment of a chromosome's layup. Then GA comes to its second phase. During this phase, the GA already found proper individuals which could satisfy the constraint, so the target in this stage is to improve fitness. This means GA needs to adjust its inner structure, at the point 2 and 3 on the generation-fitness curve, the fitness curves go up, and the corresponding strength ratio of these two points slightly go down, but both of them still satisfy the constraint.

Table III shows the searching results after conducting this experiment one hundred times in two length mutation coefficient cases for glass-epoxy and graphite-epoxy material, respectively. The best, worst case, and average experiment results are showed in this table. For the glass-epoxy material, if the length mutation coefficient takes one, the best and worst sequences are $\left[0_{40} / 90_{26}\right]_{s},\left[90_{24} / 0_{38} / \overline{90}\right]_{s}$; the average mass, cost, and the number of layers are $7.83,123,123$. If we increase its length mutation coefficient, suppose it is five, the number of layers for best and worst cases are 125 and 136; the average mass, cost, and number of layers are 8.55, 131,131 . When graphite-epoxy is taken as the experiment

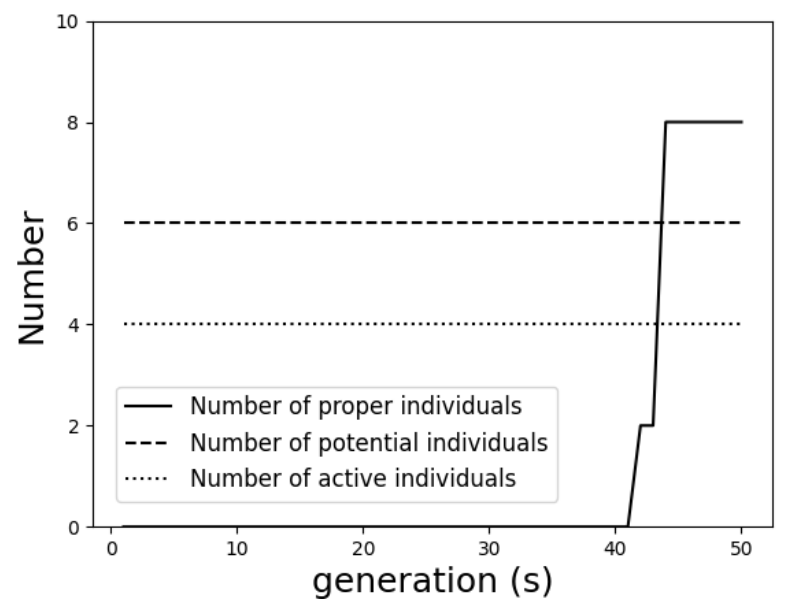

Fig. 7. Number of Individuals in Each Group as a Function of Generation. b

TABLE III. THE OPTIMUM LAYUP FOR THE LOADING $N_{x}=1 e^{6} \mathrm{~N}$ WHEN Changing the Length Mutation Coefficient, the Performance OF THE GA CAN BE IMPROVED WHEN THE LENGHT MUTATION COEFFICIENT IS SMALLER.

\begin{tabular}{|c|c|c|c|c|c|c|c|}
\hline $\begin{array}{l}\text { Length mutation } \\
\text { coefficient }\end{array}$ & Material & case & Stacking sequence & Strength ratio & Mass & Cost & Layer \\
\hline \multirow{6}{*}{1} & \multirow{3}{*}{ glass-epoxy } & worst & {$\left[0_{40} / 90_{26}\right]$} & 2.010 & 8.58 & 132 & 132 \\
\hline & & best & {$\left[90_{24} / 0_{38} / \overline{90}\right]_{s}$} & 2.078 & 8.12 & 125 & 125 \\
\hline & & average & & 2.012 & 7.83 & 123 & 123 \\
\hline & \multirow{4}{*}{ graphite-epoxy } & worst & {$\left[0_{9} / 90_{4} / \overline{0}\right]$} & 2.17 & 1.41 & 68 & 27 \\
\hline & & best & {$\left[0_{9} / 90_{1} / \overline{0}\right]$} & 2.15 & 1.10 & 53 & 21 \\
\hline & & average & & 2.018 & 1.47 & 70 & 28 \\
\hline \multirow{6}{*}{5} & & worst & {$\left[0_{36} / 90_{32}\right]_{s}$} & 2.009 & 8.84 & 136 & 136 \\
\hline & \multirow{3}{*}{ glass-epoxy } & best & {$\left[0_{36} / 90_{26} / 90\right]_{s}$} & 2.003 & 8.12 & 125 & 125 \\
\hline & & average & & 2.008 & 8.55 & 131 & 131 \\
\hline & & worst & {$\left[0_{9} / 90_{12}\right]_{s}$} & 2.006 & 2.20 & 105 & 42 \\
\hline & \multirow{2}{*}{ graphite-epoxy } & best & {$\left[00_{8} / 90_{3} / 0\right]_{s}$} & 2.001 & 1.20 & 57 & 23 \\
\hline & & average & & 2.022 & 1.54 & 73 & 29 \\
\hline
\end{tabular}

material, similar experiment results are found. Comparing these two results, we see that a bigger value of length mutation coefficient can improve this GA's performance. This is because the mutation coefficient can control both the convergence speed and search performance, a small mutation coefficient would slow the convergence speed, however, it would lead to smallgrained exploitation in the local space.

Table IV shows the optimal cross-ply sequences by the proposed GA and Choudhury and Mondal's [30] study. For the loading case $N x=1 \mathrm{MPa} \mathrm{m}$, the optimal layups are a $\left[0_{68} / 90_{72}\right]$ cross-ply laminate if glass-epoxy is taken; however, in the present study, a $\left[90_{24} / 0_{38} / 90\right]_{s}$ glass-epoxy crossply laminate is found which significantly reduces both the cost and weight, and it satisfies the constraint. Similarly, if graphite-epoxy is taken, compared with the $\left[0_{17} / 90_{18}\right]$ crossply laminate, an alternative solution is found, its layup is $\left[0_{9} / 90_{1} / \overline{0}\right]_{s}$. For both cases, we can see that the experiment results show that using the present proposed GA can obtain better results.

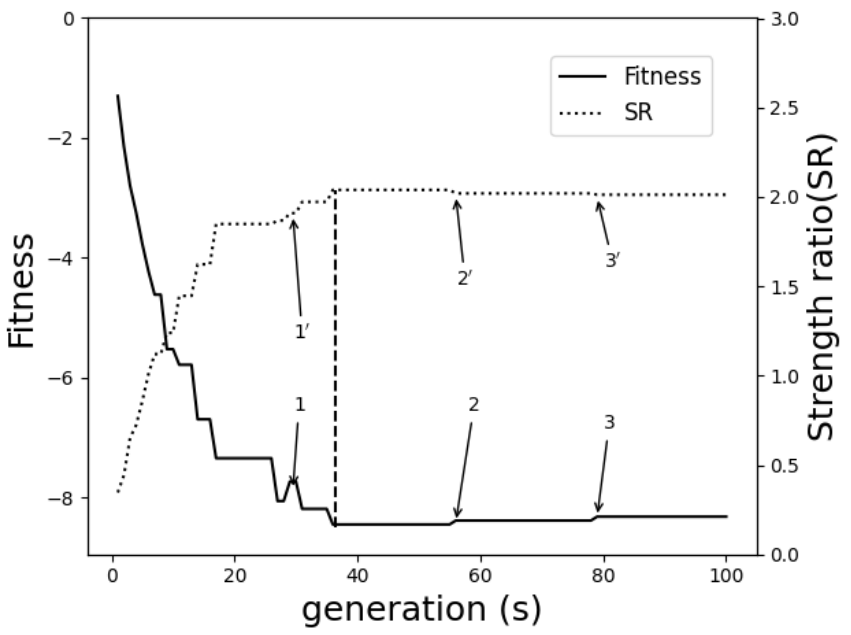

Fig. 8. The Fitness is the Negation of the Individual's Mass. The Solid Curve is the Fitness of the Best Individual in the Population in Respect to the Generations; and the Dotted Line Denotes its Corresponding Strength Ratio. If no Individuals in the Population Satisfy the Constraint, the best Individual is the one with the Biggest Strength Ratio; if not, the best Individual is the one with the Smallest Mass. 
TABLE IV. COMPARISON OF EXPERIMENT RESULTS OF CHOUDHURY AND MONDAL'S[30] AND CURRENT STUDY UNDER IN-PLANE LOADING $N_{x}=1 e 6 \mathrm{~N}$. The Results of PRESENT STUdy IS FROM PREVIOUS EXPERIMENT.

\begin{tabular}{c|cc|cc}
\hline Cross Ply $\left[0_{M} / 90_{N}\right]$ & \multicolumn{2}{c}{ Choudhury and Mondal's study } & \multicolumn{2}{c}{ Present study } \\
\hline Material & Glass-Epoxy & Graphite-Epoxy & Glass-Epoxy & Graphite-Epoxy \\
M & 68 & 17 & 76 & 19 \\
N & 72 & 18 & 49 & 2 \\
no. of lamina(n) & 140 & 35 & 125 & 21 \\
SR & 2.01 & 2.10 & 2.078 & 2.15 \\
weight & 9.10 & 1.84 & 8.12 & 1.10 \\
cost & 140 & 87.5 & 125 & 53 \\
\hline
\end{tabular}

\section{CONCLUSIONS}

In this paper, we reviewed the use of the proposed genetic algorithm framework, classical lamination theory, and TsaiWu failure theory for the layup design for cross-ply laminate under different loading cases. The principal applications of this genetic algorithm are in the design of composite laminate material with imposed constraints.

The main contribution of the present work is the genetic algorithm framework for constrained problems since the traditional genetic algorithm is primarily concerned with solving the unconstrained problem, which is not suitable for a constrained case. We deal with these constrained problems by the use of mixing strategies of selection methods instead of adding any punishment terms into the objective function. The performance of this algorithm is heavily influenced by the coefficient of the length mutation. Both for the glassepoxy and graphite-epoxy material cases, if the coefficient takes a relatively low value, this algorithm can obtain better results than when the coefficient value is high. However, The algorithm converged more quickly with a high coefficient value of length mutation.

This variant of the genetic algorithm provides a new approach to address the constrained search, and this method can be easy to apply in other domains. A drawback of the proposed genetic algorithm is more parameters involving in this GA, which makes fine-tuning more difficult.

\section{ACKNOWLEDGMENT}

The paper was supported by China Scholarship Council with the code number 201806630112

\section{REFERENCES}

[1] R. L. Riche and R. T. Haftka, "Optimization of laminate stacking sequence for buckling load maximization by genetic algorithm," AIAA journal, vol. 31, no. 5, pp. 951-956, 1993.

[2] S. Nagendra, D. Jestin, Z. Gürdal, R. T. Haftka, and L. T. Watson, "Improved genetic algorithm for the design of stiffened composite panels," Computers \& Structures, vol. 58, no. 3, pp. 543-555, 1996.

[3] D. Sadagopan and R. Pitchumani, "Application of genetic algorithms to optimal tailoring of composite materials," Composites Science and Technology, vol. 58, no. 3-4, pp. 571-589, 1998.

[4] A. Todoroki and R. T. Haftka, "Stacking sequence optimization by a genetic algorithm with a new recessive gene like repair strategy," Composites Part B: Engineering, vol. 29, no. 3, pp. 277-285, 1998.

[5] B. Liu, R. T. Haftka, M. A. Akgün, and A. Todoroki, "Permutation genetic algorithm for stacking sequence design of composite laminates," Computer methods in applied mechanics and engineering, vol. 186, no. 2-4, pp. 357-372, 2000.

[6] K. Sivakumar, N. Iyengar, and K. Deb, "Optimum design of laminated composite plates with cutouts using a genetic algorithm," Composite Structures, vol. 42, no. 3, pp. 265-279, 1998
[7] M. Walker and R. E. Smith, "A technique for the multiobjective optimisation of laminated composite structures using genetic algorithms and finite element analysis," Composite structures, vol. 62, no. 1, pp. 123-128, 2003.

[8] C.-C. Lin and Y.-J. Lee, "Stacking sequence optimization of laminated composite structures using genetic algorithm with local improvement," Composite structures, vol. 63, no. 3-4, pp. 339-345, 2004.

[9] J.-H. Kang and C.-G. Kim, "Minimum-weight design of compressively loaded composite plates and stiffened panels for postbuckling strength by genetic algorithm," Composite structures, vol. 69, no. 2, pp. 239246, 2005.

[10] M. Murugan, S. Suresh, R. Ganguli, and V. Mani, "Target vector optimization of composite box beam using real-coded genetic algorithm: a decomposition approach," Structural and Multidisciplinary Optimization, vol. 33, no. 2, pp. 131-146, 2007.

[11] M. Akbulut and F. O. Sonmez, "Optimum design of composite laminates for minimum thickness," Computers \& Structures, vol. 86, no. 21-22, pp. 1974-1982, 2008.

[12] C. R. Houck, J. Joines, and M. G. Kay, "A genetic algorithm for function optimization: a matlab implementation," Ncsu-ie tr, vol. 95, no. 09, pp. $1-10,1995$.

[13] M. D. Vose, The simple genetic algorithm: foundations and theory. MIT press, 1999.

[14] M. Zbigniew, "Genetic algorithms+ data structures= evolution programs," Computational Statistics, pp. 372-373, 1996.

[15] O. Seresta, Z. Gürdal, D. B. Adams, and L. T. Watson, "Optimal design of composite wing structures with blended laminates," Composites Part B: Engineering, vol. 38, no. 4, pp. 469-480, 2007.

[16] G. Soremekun, Z. Gürdal, R. Haftka, and L. Watson, "Composite laminate design optimization by genetic algorithm with generalized elitist selection," Computers \& structures, vol. 79, no. 2, pp. 131-143, 2001.

[17] R. Le Riche and R. Haftka, "Improved genetic algorithm for minimum thickness composite laminate design," Composites Engineering, vol. 5, no. 2, pp. 143-161, 1995.

[18] A. Y. Abu-Odeh and H. L. Jones, "Optimum design of composite plates using response surface method," Composite structures, vol. 43 , no. 3 , pp. 233-242, 1998.

[19] C. Fang and G. S. Springer, "Design of composite laminates by a monte carlo method," Journal of composite materials, vol. 27, no. 7, pp. 721753, 1993.

[20] D. J. Deka, G. Sandeep, D. Chakraborty, and A. Dutta, "Multiobjective optimization of laminated composites using finite element method and genetic algorithm," Journal of reinforced plastics and composites, vol. 24, no. 3, pp. 273-285, 2005.

[21] C. H. Park, W. I. Lee, W. S. Han, and A. Vautrin, "Improved genetic algorithm for multidisciplinary optimization of composite laminates," Computers \& structures, vol. 86, no. 19-20, pp. 1894-1903, 2008.

[22] S. Omkar, R. Khandelwal, S. Yathindra, G. N. Naik, and S. Gopalakrishnan, "Artificial immune system for multi-objective design optimization of composite structures," Engineering Applications of Artificial Intelligence, vol. 21, no. 8, pp. 1416-1429, 2008.

[23] J.-S. Kim, "Development of a user-friendly expert system for composite laminate design," Composite Structures, vol. 79, no. 1, pp. 76-83, 2007.

[24] M. Gholami, A. Fathi, and A. M. Baghestani, "Multi-objective optimal structural design of composite superstructure using a novel monmpso algorithm," International Journal of Mechanical Sciences, p. 106149, 2020.

[25] T. N. Massard, "Computer sizing of composite laminates for strength," Journal of reinforced plastics and composites, vol. 3, no. 4, pp. 300345, 1984.

[26] J. Reddy and A. Pandey, "A first-ply failure analysis of composite laminates," Computers \& Structures, vol. 25, no. 3, pp. 371-393, 1987.

[27] A. Soeiro, C. C. António, and A. T. Marques, "Multilevel optimization of laminated composite structures," Structural optimization, vol. 7, no. 1-2, pp. 55-60, 1994.

[28] J. L. Pelletier and S. S. Vel, "Multi-objective optimization of fiber reinforced composite laminates for strength, stiffness and minimal mass," Computers \& structures, vol. 84, no. 29-30, pp. 2065-2080, 2006.

[29] P. Jadhav and P. R. Mantena, "Parametric optimization of grid-stiffened composite panels for maximizing their performance under transverse loading," Composite structures, vol. 77, no. 3, pp. 353-363, 2007. 
[30] A. Choudhury, S. Mondal, and S. Sarkar, "Failure analysis of laminated composite plate under hygro-thermo mechanical load and optimisation," International Journal of Applied Mechanics and Engineering, vol. 24 no. 3, pp. 509-526, 2019.

[31] P. Martin, "Optimum design of anisotropic sandwich panels with thin faces," Engineering optimization, vol. 11, no. 1-2, pp. 3-12, 1987.

[32] C. M. Soares, V. F. Correia, H. Mateus, and J. Herskovits, "A discrete model for the optimal design of thin composite plate-shell type structures using a two-level approach," Composite structures, vol. 30, no. 2, pp. 147-157, 1995.

[33] R. Watkins and A. Morris, "A multicriteria objective function optimization scheme for laminated composites for use in multilevel structura optimization schemes," Computer Methods in Applied Mechanics and Engineering, vol. 60, no. 2, pp. 233-251, 1987.

[34] G. N. Naik, S. Gopalakrishnan, and R. Ganguli, "Design optimization of composites using genetic algorithms and failure mechanism based failure criterion," Composite Structures, vol. 83, no. 4, pp. 354-367, 2008. 